\title{
Truth-Telling and Nash Equilibria in Minimum Cost Spanning Tree Models
}

\author{
Jens Leth Hougaard* Mich Tvede ${ }^{\dagger}$
}

\begin{abstract}
In this paper we consider the Minimum Cost Spanning Tree model. We assume that a central planner aims at implementing a minimum cost spanning tree not knowing the true link costs. The central planner sets up a game where agents announce link costs, a tree is chosen and costs are allocated according to the rules of the game. We characterize ways of allocating costs such that true announcements constitute Nash equilibria both in case of full and incomplete information. In particular, we find that the Shapley rule based on the irreducible cost matrix is consistent with truthful announcements while a series of other well-known rules (such as the Bird-rule, Serial Equal Split, the Proportional rule etc.) are not.
\end{abstract}

Keywords: Bayesian Nash equilibrium, Incomplete information, Minimum cost spanning tree, Shapley value, Nash equilibrium, Truth-telling.

Acknowledgements: The authors wish to thank Gustavo Bergantinos, Hervé Moulin, Kurt Nielsen and Katrine Stagaard for helpful comments.

\footnotetext{
${ }^{*}$ Corresponding author: Institute of Food and Resource Economics, University of Copenhagen, Rolighedsvej 25, DK-1958 Frederiksberg C., Denmark, phone +453533 68 14, email: jlh@foi.dk

${ }^{\dagger}$ Department of Economics, University of Copenhagen, Oester Farimagsgade 5, DK1353 Copenhagen K, Denmark, phone +45 353230 92, email: mich.tvede@econ.ku.dk
} 


\section{Introduction}

Recently, economists have shown a growing interest in networks and the literature is becoming rich on various issues and models, see e.g. Goyal (2007) and Jackson (2008). In the present paper we consider the relation between cost allocation and efficient network structure within the classical Minimum Cost Spanning Tree (MCST) model. Here, a group of agents is to be connected to a source (supplier) in the least costly way and face the problem of sharing the cost of the efficient network, see e.g. Sharkey (1995) - practical examples include district heating, computer network using a common server, cable tv, chain stores using a common warehouse etc.

While the literature typically contains axiomatic analysis and comparisons of different cost sharing methods there has been less emphasis on strategic issues concerning practical implementation. Clearly, agents can have private information about the cost structure. This information they can use strategically to lower their own cost at the expense of a loss in social efficiency.

It is well-known that in general we cannot find incentive compatible and efficient cost allocation mechanisms (Green \& Laffont 1977), but under special circumstances such mechanisms can in fact be constructed, see e.g. Jackson \& Moulin (1992), Schmeidler \& Tauman (1994), Young (1998), Moulin \& Shenker (2001). For social choice rules Maskin (1999) and Dasgupta, Hammond \& Maskin (1979) show that implementability and monotonicity are equivalent. We consider monotonicity of cost allocation rules and present compatible results for the MCST model.

In the specific context of the MCST model, implementation has been analyzed in a few recent papers; Bergantinos \& Lorenzo (2004, 2005) and Bergantinos \& Vidal-Puga (2010). All three papers consider existence and properties of Nash equilibria and subgame perfect Nash equilibria of noncooperative sequential bargaining procedures. The first two papers study a real life allocation problem where agents sequentially join an existing network along the lines of the Prim algorithm (Prim 1957). The latter paper shows that another Prim-like procedure, where agents announce their willingness to pay for other agents to connect to the source, leads to a unique subgame 
perfect Nash equilibrium in which costs are allocated corresponding to the use of the Shapley value on the related irreducible cost matrix (dubbed the Folksolution in Bogomolnaia \& Moulin 2010 and further analyzed in Bergantinos \& Videl-Puga 2007, 2009 and Hougaard et al. 2010).

In the present paper, Section 2 briefly reviews the MCST model and Section 3 introduces a noncooperative game form involving a planner who does not know the link costs and a set of agents who all know the link costs. First the planner announces the rules of the game being an allocation rule and an estimation rule. Then agents announce the link costs, which in turn are used to estimate a cost matrix and its related set of MCSTs. A particular MCST is selected at random and the realized link costs are shared between the agents according to the announced allocation rule.

Compared to the Prim-like sequential mechanisms mentioned above our approach is different since it is a simple one-shot game, which is not based on any algorithm for finding the MCST. Moreover, agents' announcements do not directly influence their cost shares since these are determined by the realized costs along the chosen spanning tree.

In Section 4 our main result is established: announcing the true link costs constitutes a Nash equilibrium if and only if the associated allocation rule is monotonic (in the sense that cost shares are weakly increasing in the irreducible cost matrix). Consequently, monotonic allocation rules such as the Shapley rule (on the irreducible cost matrix) and the Equal Split rule will both result in truth-telling Nash equilibria where the planner can implement the true MCST. However, well-known rules such as the Bird rule and the Proportional rule fail to satisfy monotonicity.

In Section 5 we consider an incomplete information version of the game along the lines of Jackson (1991). We show that truth-telling remains an equilibrium for monotonic allocation rules. Section 6 closes with some final remarks.

\section{The MCST Model}

Recall the minimum cost spanning tree model (see e.g. Sharkey 1995). Networks, where a source supplies agents with some homogeneous good, are 
considered. Let 0 be the source and let $N=\{1, \ldots, n\}$ be the set of agents. A network $g$ over $N^{0}=\{0\} \cup N$ is a set of unordered pairs $a b$, where $a, b \in N^{0}$. Let $N^{0}(2)$ be the set of unordered pairs and let $G^{0}=\left\{g \mid g \subset N^{0}(2)\right\}$ be the set of all networks over $N^{0}$.

In a network $g$ two agents $a$ and $b$ are connected if and only if there is a path $i_{1} i_{2}, i_{2} i_{3}, \ldots, i_{m-1} i_{m}$ such that $i_{h} i_{h+1} \in g$ for $1 \leq h \leq m-1$ where $i_{1}=a$ and $i_{m}=b$. A network $g$ is connected if $a$ and $b$ are connected for all $a, b \in N^{0}$. A path is a cycle if it starts and ends with the same agent. A network is a tree if it contains no cycles. A spanning tree is a tree where all agents in $N^{0}$ are connected. There are $(n+1)^{n-1}$ spanning trees.

For every pair of agents $a b \in N^{0}(2)$ there is a net-cost $k_{a b} \in \mathbb{R}$ attached to the link between $a$ and $b$. If $k_{a b}>(<) 0$, then there is a net-cost (net-benefit) of establishing the link. For $N^{0}$ let the $(n+1) \times(n+1)$-matrix $K$ be the net-cost matrix. The model is a slight generalization of the standard MCST model because link costs can be positive as well as negative. An allocation problem is a set of agents and a net-cost matrix, $(N, K)$.

For a spanning tree $p$, let $v(N, K, p)=\sum_{a b \in p} k_{a b}$ be the total net-cost of $p$. A minimum cost spanning tree (MCST) is a spanning tree $p$ such that $v(N, K, p) \leq v(N, K, q)$ for every spanning tree $q$. For every allocation problem $(N, K)$ there exists a MCST because the number of spanning trees is finite. Let $v(N, K)$ denote the minimal cost so there exists a spanning tree $p$ such that $v(N, K, p)=v(N, K)$ and $v(N, K, q) \geq v(N, K)$ for every spanning tree $q$. If all net-costs $k_{a b}$ are different then there is a unique MCST, but in general there can be several MCSTs. Indeed, if all net-costs $k_{a b}$ are equal, then every spanning tree is a MCST. Let $T(N, K)$ be the set of MCSTs.

\section{Irreducible matrices}

For two matrices $K$ and $K^{\prime}$ the matrix $K$ is smaller than $K^{\prime}$ if and only if $k_{a b} \leq k_{a b}^{\prime}$ for all $a, b \in N^{0}$. The irreducible matrix $C(K)$ for a net-cost matrix $K$ is the smallest matrix $C$ such that $v(N, C)=v(N, K)$ and $c_{a b} \leq k_{a b}$ for all $a, b \in N^{0}$, see e.g. Bird (1976), Aarts \& Driessen (1993). For a net-cost matrix $K$ and a spanning tree $p$ the irreducible matrix $C(K, p)$ is defined as follows: For every $a, b \in N^{0}$, let $p_{a b}$ be the unique path in $p$ from $a$ to $b$, 
then $c_{a b}=\max _{i j \in p_{a b}}\left\{k_{i j}\right\}$. It is known that if $p^{*}$ is a MCST, then $C(K)=$ $C\left(K, p^{*}\right)$. Hence if $p^{*}$ and $q^{*}$ are MCSTs, then $C\left(K, p^{*}\right)=C\left(K, q^{*}\right)=C(K)$, and if $p^{*}$ is a MCST and $p$ is spanning tree, then $C\left(K, p^{*}\right)$ is smaller than $C(K, p)$.

\section{Allocation Rules}

Let $\Gamma$ be the set of allocation problems and their spanning trees so $(N, K, p) \in$ $\Gamma$ if and only if $(N, K)$ is an allocation problem and $p$ is a spanning tree for $(N, K)$. An allocation rule $\phi: \Gamma \rightarrow \mathbb{R}^{N}$ maps an allocation problem $(N, K)$ and a spanning tree $p$ to an $n$-dimensional vector of net-costs $\phi(N, K, p)=$ $\left(\phi_{1}(N, K, p), \ldots, \phi_{n}(N, K, p)\right)$.

Only allocation rules that are budget balanced, reductionist ${ }^{1}$ and continuous are considered.

- Budget-balance: $\sum_{a \in N} \phi_{a}(N, K, p)=v(N, K)$ for all $p \in T(N, K)$, so cost shares add up to the total cost of the MCST.

- Reductionist: $\phi_{a}(N, K, p)=\phi_{a}(N, C(K, p), p)$ for all spanning trees $p$, so cost shares depend on the irreducible matrix of the chosen spanning tree.

- Continuity: $\phi(N, K, p)$ is continuous in $K$.

Budget-balance and continuity are standard properties of allocation rules. Budget-balance implies that exactly the net-cost of every spanning tree is allocated. The reason we consider reductionist rules will become clear once the implementation setting is presented.

For a spanning tree $p$, let $\delta(a, b, p)$ be the unique neighbor of $a$ in the path $p_{a b}$ from $a$ to $b$ in $p$, then some examples of (continuous, budget-balanced and reductionist) allocation rules are:

- The Equal Split Rule: For all $a \in N$

$$
\phi_{a}^{E}(N, K, p)=\frac{v(N, C(K, p))}{n} .
$$

\footnotetext{
${ }^{1}$ Allocation rules based on the irreducible matrix are denoted reductionist rules in Bogomolnaia \& Moulin (2010).
} 
- The Bird Rule: For all $a \in N$

$$
\phi_{a}^{B}(N, K, p)=c_{a b}(K, p)
$$

where $b=\delta(a, 0, p)$

- The Proportional Rule: For all $i \in N$

$$
\phi_{a}^{P}(N, K, p)=\frac{c_{0 a}(K, p)}{\sum_{b \in N} c_{0 b}(K, p)} v(N, C(k, p)) .
$$

- The Shapley Rule: For all $a \in N$

$$
\phi_{a}^{S}(N, K, p)=\sum_{S \subset N: a \in S} \frac{(s-1) !(n-s) !}{n !}(v(S, C(K, p))-v(S \backslash a, C(K, p)))
$$

where $s$ is the number of agents in $S$ and $v(S, C(K, p))$ is the minimal cost of connecting all members of $S$ to the source 0 given $C(K, p){ }^{2}$

\section{The Game}

In this section a two-stage game between a central planner and a set of agents is introduced. The planner aims at minimizing the cost of connecting all agents to the source, but the planner does not know the link costs. Every agent aims at minimizing his connection cost and knows all link costs.

The game is as follows: first, the planner announces the rules of the game, i.e. an estimation rule and an allocation rule. Then every agent announces (simultaneously and independently) all link costs to the planner who use the estimation rule to estimate the cost matrix based on these announcements and finds the associated set of MCSTs. Second, the planner randomly selects an efficient spanning tree among this set and uses the allocation rule to distribute the observed (true) costs of the selected spanning tree to the agents.

The announcements are used to select a spanning tree because the announcements are used to estimate link costs. However, the announcements are not used to distribute observed costs.

\footnotetext{
${ }^{2}$ In our context the Shapley value coincides with the so-called Folk-solution considered in Bergantinos \& Vidal-Puga (2007, 2009) and Bogomolnaia \& Moulin (2010).
} 
To be more precise, the rules of the game consists of an allocation rule $\phi: \Gamma \rightarrow \mathbb{R}^{n}$ and an estimation rule $\tau: \mathbb{R}^{n} \rightarrow \mathbb{R}$. Let $\sigma \in\left(\mathbb{R}^{N^{0}(2)}\right)^{n}$, where $\sigma=\left(\sigma^{1}, \ldots, \sigma^{n}\right)$ and $\sigma^{a}=\left(\sigma_{01}^{a}, \ldots, \sigma_{n-1 n}^{a}\right)$ for all $a$, be a list of individual announcements of all link costs. The estimation rule $\tau$ is supposed to have values between the minimum and maximum announcement so $\tau\left(\sigma_{a b}^{1}, \ldots, \sigma_{a b}^{n}\right) \in\left[\min \left\{\sigma_{a b}^{1}, \ldots, \sigma_{a b}^{n}\right\}, \max \left\{\sigma_{a b}^{1}, \ldots, \sigma_{a b}^{n}\right\}\right]$, and to be upward unbounded so $\lim _{\sigma_{a b}^{z} \rightarrow \infty} \tau\left(\sigma_{a b}^{1}, \ldots, \sigma_{a b}^{n}\right)=\infty$ for every $z \in N$.

For a list of individual announcements of link costs $\sigma$ the estimated cost matrix is $K^{e}(\sigma)$, where $k_{a b}^{e}=\tau\left(\sigma_{a b}^{1}, \ldots, \sigma_{a b}^{n}\right)$ for all $a, b$. For every $p \in$ $T\left(N, K^{e}(\sigma)\right)$ the cost shares are $\left(\phi_{1}(N, C(K, p), p), \ldots, \phi_{1}(N, C(K, p), p)\right)$. Every $p \in T\left(N, K^{e}(\sigma)\right)$ is equally likely, so the average cost of agent $a$ is

$$
\sum_{p \in T\left(N, K^{e}(\sigma)\right)} \frac{\phi_{a}(N, C(K, p), p)}{\left|T\left(N, K^{e}(\sigma)\right)\right|} .
$$

For fixed rules of the game agents can choose their announcements strategically. Indeed, by lying an agent can influence the set of efficient spanning trees given the estimated link costs and thereby manipulate his resulting cost share.

Example: Consider the Proportional Rule $\phi^{P}$. Let $N=\{1,2,3\}$ and let

$$
K=\left(k_{01}, k_{02}, k_{03}, k_{12}, k_{13}, k_{23}\right)=(1,10,10,2,3,0) .
$$

Then $p^{*}=(01,12,23)$ is the unique MCST and $C(K)=(1,2,2,2,2,0)$ so $\phi_{1}^{P}\left(N, C\left(K, p^{*}\right), p^{*}\right)=3 / 5$. Suppose that agents 2 and 3 announce the true link costs and let the estimation rule be the average, then agent 1 can gain by announcing $\sigma_{12}^{1}>5$ and the true link costs for the other links. Then $q^{*}=(01,13,32)$ is the unique MCST, $K^{e}=\left(1,10,10, k_{12}^{e}, 3,0\right)$, where $k_{12}^{e}>3$, and $C\left(K, q^{*}\right)=(1,3,3,3,3,0)$ so $\phi_{1}^{P}\left(N, C\left(K, q^{*}\right), q^{*}\right)=4 / 7<3 / 5$.

End of example

The example indicates that some form of monotonicity of the allocation rules is crucial for truth-telling.

(M) Monotonicity: An allocation rule is monotonic provided that for two spanning trees $p$ and $q$, if $C(K, p)$ is smaller than $C(K, q)$, then

$$
\phi_{a}(N, C(K, p), p) \leq \phi_{a}(N, C(K, q), q)
$$


for all $a \in N$.

Neither the Bird rule nor the Proportional rule satisfy monotonicity. However, both the Equal Split rule and the Shapley rule satisfies monotonicity. In fact, the set of monotonic rules is quite large and also includes, for instance, the family of obligation rules introduced and analyzed in Tijs et al. (2006).

\section{Implementation of MCSTs}

The relation between truth-telling as a strategy and monotonic allocation rules is characterized below.

Theorem 1 Truth-telling is a Nash equilibrium for every allocation problem if and only if the allocation rule is monotonic.

Proof: Suppose that an allocation rule is monotonic. Then no agent can gain by deviating from truth-telling, because the irreducible matrix is minimal for truth-telling.

Suppose that an allocation rule is not monotonic. Then there exists an allocation problem $(N, K)$ and spanning trees $p$ and $q$ such that $C(K, q) \geq$ $C(K, p)$ and $\phi_{z}(N, C(K, q), q)<\phi_{z}(N, C(K, p), p)$ for some $z \in N$. Let a new problem $\left(N, K^{\prime}\right)$ be defined by $k_{a b}^{\prime}=k_{a b}$ for all $a b \in p, k_{a b}^{\prime}=k_{a b}+\varepsilon$ for $a b \in q \backslash p$ for $\varepsilon>0$ and $k_{a b}^{\prime}=\max _{a^{\prime} b^{\prime}}\left\{k_{a^{\prime} b^{\prime}}\right\}+1$ otherwise. Then $p$ is the unique MCST in $\left(N, K^{\prime}\right)$ and $\phi_{z}\left(N, K^{\prime}, q\right)<\phi_{z}\left(N, K^{\prime}, p\right)$ by continuity for $\varepsilon$ sufficiently small. Suppose that all agents except $z$ announce the truth. Then $z$ can gain by announcing the truth for all links in $q$ and sufficiently high costs for all other links. Indeed $q$ is the unique MCST for $\left(N, K^{e}\right)$ because $\tau$ is upward unbounded.

Q.E.D.

Remark: The theorem remains valid if the notion of equilibrium is strengthened from Nash equilibrium to strong Nash equilibrium.

End of remark

The relation between Nash equilibria and implementation of MCSTs is characterized below. 
Theorem 2 Suppose that $\sigma$ is a Nash equilibrium. If $p \in T\left(N, K^{e}(\sigma)\right)$, then $p \in T(N, K)$.

Proof: Suppose that $\sigma$ is a Nash equilibrium and $p \in T\left(N, K^{e}(\sigma)\right)$ but $p \notin T(N, K)$. Then

$$
\sum_{a \in N} \sum_{p \in T\left(N, K^{e}(\sigma)\right)} \frac{\phi_{a}(N, K, p)}{\left|T\left(N, K^{e}(\sigma)\right)\right|}>\sum_{a \in N} \sum_{q \in T(N, K)} \frac{\phi_{a}(N, K, q)}{|T(N, K)|} .
$$

Therefore

$$
\sum_{p \in T\left(N, K^{e}(\sigma)\right)} \frac{\phi_{z}(N, K, p)}{\left|T\left(N, K^{e}(\sigma)\right)\right|}>\phi_{z}(N, K, q),
$$

for some $z$ and $q \in T(N, K)$. Then $z$ can gain by announcing the true link costs for all links in $q$ and sufficiently high costs for all other links. Indeed $q$ is the unique MCST for $\left(N, K^{e}\right)$ because $\tau$ is upward unbounded. This contradicts that $\sigma$ is a Nash equilibrium.

Q.E.D.

The proofs of Theorems 1 and 2 demonstrate the importance of lies. In both proofs an agent manipulates the estimated link costs by lying about non-realized link costs showing that attempts by the planner to enforce truthtelling by punishing observed lies are in vain.

Next we demonstrate that Theorem 1 generalizes to situations where agents have incomplete information about costs, while Theorem 2 does not.

\section{Incomplete information}

Let $\left\{K_{1}, \ldots, K_{m}\right\}$ be a finite set of cost matrices known by all agents. Every agent $a \in N$ has a probability measure on the finite set of cost matrices $\mu_{a}:\left\{K_{1}, \ldots, K_{m}\right\} \rightarrow[0,1]$ with $\mu_{a}(K)>0$ for all $K \in\left\{K_{1}, \ldots, K_{m}\right\}$. In addition every agent $a \in N$ has a signal from the finite set of cost matrices to subsets of that set $s_{a}:\left\{K_{1}, \ldots, K_{m}\right\} \rightarrow 2^{\left\{K_{1}, \ldots, K_{m}\right\}}$ where $s_{a}(K)$ is the private information of agent $a \in N$ at $K$. For signals it is assumed that: 1. if the true matrix is $K$, then $K$ is part of the private information at $K$; 2. the matrix $K^{\prime}$ is part of the private information at $K$ if and only if $K$ is part of the private information at $K^{\prime}$; and, 3 . the intersection of all private 
information sets consists of the true matrix. Formally: $1 . K \in s_{a}(K) ; 2$. $K^{\prime} \in s_{a}(K)$ if and only if $K \in s_{a}\left(K^{\prime}\right)$; and, 3. $\cap_{a} s_{a}(K)=\{K\}$.

An allocation problem with incomplete information consists of a set of agents, a finite set of cost matrices and a list of individual signals and probability measures, $\left(N,\left\{K_{1}, \ldots, K_{m}\right\},\left(\mu_{a}, s_{a}\right)_{a}\right)$.

The planner is ignorant in the sense that he knows neither the set of cost matrices $\left\{K_{1}, \ldots, K_{m}\right\}$ nor the probability measures and signals of the agents $\left(s_{a}, \mu_{a}\right)_{a}$.

Let $\mathbb{K}$ be the set of all cost matrices and $\mathbb{F}$ the set of all finite subsets of $\mathbb{K}$. The planner chooses a mechanism that consists of an announcement set for every agent, a cost allocation rule and an estimation rule. The announcement set of agent $a$ is the set $\mathbb{F}$. The cost allocation rule is a map $\phi: \Gamma \rightarrow \mathbb{R}^{n}$. The estimation rule is a map $K^{e}:(\mathbb{F})^{n} \rightarrow \mathbb{K}$ where $K^{e}(\sigma) \in \operatorname{co}\left(\cap_{a} \sigma_{a}\right)$ if $\cap_{a} \sigma_{a} \neq \emptyset$ and $k_{a^{\prime} b^{\prime}}^{e}(\sigma) \rightarrow \infty$ if $\max _{a} \min _{K \in \sigma_{a}} k_{a^{\prime} b^{\prime}} \rightarrow \infty$.

If the cost matrix is $K$ and the list of individual announcements is $\sigma$, then the average cost of agent $a$ is

$$
\sum_{p \in T\left(N, K^{e}(\sigma)\right)} \frac{1}{\left|T\left(N, K^{e}(\sigma)\right)\right|} \phi_{a}(N, K, p)
$$

and the expected cost of agent $a$ is

$$
\sum_{K^{\prime} \in s_{a}(K)} \frac{\mu_{a}\left(K^{\prime}\right)}{\sum_{K^{\prime \prime} \in s_{a}(K)} \mu_{a}\left(K^{\prime \prime}\right)} \sum_{p \in T\left(N, K^{e}(\sigma)\right)} \frac{1}{\left|T\left(N, K^{e}(\sigma)\right)\right|} \phi_{a}\left(N, K^{\prime}, p\right) .
$$

For a cost allocation problem with incomplete information a strategy for agent $a$ is a map $f_{a}:\left\{K_{1}, \ldots, K_{m}\right\} \rightarrow \mathbb{F}$ that is measurable with respect to $s_{a}$, i.e., if $s_{a}\left(K^{\prime}\right)=s_{a}(K)$, then $f_{a}\left(K^{\prime}\right)=f_{a}(K)$. A Bayesian Nash equilibrium is a strategy profile such that no agent can gain be deviation.

Corollary 1 Truth-telling is a Bayesian Nash equilibrium for every allocation problem with incomplete information if and only if the allocation rule is monotonic.

Proof: Assume the allocation rule is monotonic and suppose that all agents use truth-telling as a strategy, i.e., $\sigma_{a}=s_{a}$ for all $a \in N$. Then $K^{e}(\sigma)=K$ 
so the planner is able to implement a MCST. Hence, no agent can gain by deviating.

Assume the allocation rule is not monotonic and note that cost allocation problems with full information are special cases of problems with incomplete information. Indeed allocation problems with incomplete information, where $\left\{K_{1}, \ldots, K_{m}\right\}$ is the finite set of cost matrices and $K_{m}=\ldots=K_{1}=K$ for some $K$, are equivalent to allocation problems with full information. It follows from the proof of Theorem 1 that there exists another cost allocation problem with full information where truth-telling is not a Nash equilibrium. Moreover suppose that $K$ is a cost matrix for which some agent can benefit by making a false announcement given the non-monotonic allocation rule in case of full information. Then there exists $\varepsilon>0$ such that for allocation problems with incomplete information, if $\max \left\{\left|K_{1}-K\right|, \ldots,\left|K_{m}-K\right|\right\}<\varepsilon$, then some agent can benefit by making false announcements.

Q.E.D.

Unfortunately Theorem 2 does not generalize to cost allocation problems with incomplete information as demonstrated by the following example.

Example: Let $N=\{1,2\}$ and suppose that there are four cost matrices $K=\left(k_{01}, k_{02}, k_{12}\right)$ where

$$
K_{1}=(2,3,1), K_{2}=(2,5,1), K_{3}=(4,3,1), K_{4}=(4,5,1) .
$$

Both agents learn their own link costs from their own signals so

$$
\begin{aligned}
& s_{1}(K)= \begin{cases}\left\{K_{1}, K_{2}\right\} & \text { for } K \in\left\{K_{1}, K_{2}\right\} \\
\left\{K_{3}, K_{4}\right\} & \text { for } K \in\left\{K_{3}, K_{4}\right\}\end{cases} \\
& s_{2}(K)= \begin{cases}\left\{K_{1}, K_{3}\right\} & \text { for } K \in\left\{K_{1}, K_{3}\right\} \\
\left\{K_{2}, K_{4}\right\} & \text { for } K \in\left\{K_{2}, K_{4}\right\}\end{cases}
\end{aligned}
$$

and $\mu_{a}(K)=1 / 4$ for all $K \in\left\{K_{1}, K_{2}, K_{3}, K_{4}\right\}$.

Suppose that allocation rule is monotonic and that the estimation rule is defined as follows if $\sigma_{1} \cap \sigma_{2} \neq \emptyset$, then

$$
K^{e}(\sigma)=\int_{\sigma_{1} \cap \sigma_{2}} K d K
$$


and whatever otherwise. Then the strategies $\sigma$, where

$$
\begin{gathered}
\sigma_{1}(K)=\left\{\begin{array}{cc}
\left\{K_{1}, K_{2}\right\} & \text { for } K \in\left\{K_{1}, K_{2}\right\} \\
\left\{K_{1}, K_{2}, K_{3}, K_{4}\right\} & \text { for } K \in\left\{K_{3}, K_{4}\right\}
\end{array}\right. \\
\sigma_{2}(K)=\left\{\begin{array}{cc}
\left\{K_{1}, K_{2}, K_{3}, K_{4}\right\} & \text { for } K \in\left\{K_{1}, K_{3}\right\} \\
\left\{K_{2}, K_{4}\right\} & \text { for } K \in\left\{K_{2}, K_{4}\right\},
\end{array}\right.
\end{gathered}
$$

is a Bayesian Nash equilibrium. However for $K=K_{3}$ the estimated cost matrix is $K^{e}=(3,4,1)$, but $K_{3}=(4,3,1)$ so the MCST is not implemented.

End of example

\section{Final Remarks}

For most cost allocation problems there are natural lower and upper bounds on link costs. Intuitively it appears natural to restrict announcements to be within such bounds. However if bounds are imposed on strategies, then the set of equilibria can be enlarged, possibly including strategy profiles that result in inefficient spanning trees as indirectly demonstrated in the proof of Theorem 2. In other words it is important agents are able to tell quite substantial lies.

\section{References}

[1] Aarts, H., \& T. Driessen (1993), The irreducible core of a minimum cost spanning tree game, ZOR - Methods and Models of Operations Research, $38,163-174$.

[2] Bergantinos, G., \& L. Lorenzo (2004), A non-cooperative approach to the cost spanning tree problem, Mathematical Methods of Operations Research, 59, 393-403.

[3] Bergantinos, G., \& L. Lorenzo (2005), Optimal equlibria in the noncooperative game associated with cost spanning tree problems, Annals of Operations Research, 137, 101-115. 
[4] Bergantinos, G., \& J.J. Vidal-Puga (2007), A fair rule in minimum cost spanning tree problems, Journal of Economic Theory, 137, 326-352.

[5] Bergantinos, G., \& J.J. Vidal-Puga (2010), Realizing fair outcomes in minimum cost spanning tree through non-cooperative mechanisms, European Journal of Operational Research, 201, 811-820.

[6] Bergantinos, G., \& J.J. Vidal-Puga (2009), Additivity in minimum cost spanning tree problems, Journal of Mathematical Economics, 45, 38-42.

[7] Bird, C.G., (1976), On cost allocation for a spanning tree: a game theoretic approach, Networks, 6, 335-350.

[8] Bogomolnaia, A., \& H. Moulin (2010), Sharing the cost of a minimal cost spanning tree: beyond the Folk solution, Games and Economic Behavior, 69, 238-248.

[9] Dasgupta, P., P. Hammond, P. \& E. Maskin (1979), The implementation of social choice rules: some general results on incentive compatibility, Review of Economic Studies, 46, 185-216.

[10] Goyal, S., (2007), Connections: An Introduction to the Economics of Networks, Princeton University Press.

[11] Green, J., \& J.-J. Laffont (1977), Characterization of satisfactory mechanisms for the revelation of preferences for public goods, Econometrica, 45, 427-438.

[12] Hougaard, J.L., H. Moulin \& L.P. Østerdal (2010), Decentralized pricing in minimum costs spanning trees, Economic Theory, 44, 293-306.

[13] Jackson, M., (1991), Bayesian Implementation, Econometrica, 59, 461477.

[14] Jackson, M., (2008), Social and Economic Networks, Princeton University Press.

[15] Jackson, M., \& H. Moulin (1992), Implementing a Public Project and Distributing its Cost, Journal of Economic Theory, 57, 125-40. 
[16] Maskin, E., (1999), Nash Equilibrium and Welfare Optimality, Review of Economic Studies, 66, 23-38.

[17] Moulin H., \& S. Shenker (2001), Strategyproof Sharing of Submodular Costs: Budget Balance versus Efficiency, Economic Theory, 18, 3, 511533.

[18] Prim, R.C., (1957), Shortest connection networks and some generalizations, Bell Systems Technical Journal, 36, 1389-1401.

[19] Schmeidler, D., \& Y. Tauman (1994), Incentive compatible cost allocation schemes, Journal of Economic Theory, 63, 189-207.

[20] Sharkey W.W., (1995), Network models in economics, in: Ball et al. (Eds), Handbooks in Operations Research and Management Science, Elsevier, New York, pp. 713-765.

[21] Tijs, S., R. Branzei, S. Moretti and H. Norde (2006), Obligation rules for minimum cost spanning tree situations and their monotonicity properties, European Journal of Operational Research, 175, 121-134.

[22] Young H.P., (1998), Cost allocation, demand revelation, and core implementation, Mathematical Social Sciences, 36, 213-228. 